Special issue of the 3rd International Conference on Computational and Experimental Science and Engineering (ICCESEN 2016)

\title{
Theoretical Photoneutron Cross Sections and the Effect of Gamma Strength Functions for W, Ag, Nb
}

\author{
N. KARPuZ DEMIR ${ }^{a, *}$ AND B. ÇETIN ${ }^{b}$ \\ ${ }^{a}$ Amasya University, Sabuncuoğlu Şerefeddin Vocational School of Health Services, Amasya, Turkey \\ ${ }^{b}$ Amasya University, Sciences and Arts Faculty, Amasya, Turkey
}

\begin{abstract}
In nuclear reactions the usage of high energy gamma particles, as projectiles, has some essential advantages for studying of nuclear structure and reaction mechanisms. In this work, $(\gamma, \mathrm{n})$ reaction cross sections of ${ }^{186} \mathrm{~W}$, ${ }^{107} \mathrm{Ag}$ and ${ }^{93} \mathrm{Nb}$ have been calculated with TALYS 1.6 nuclear reaction simulation code. The main aim of this study is to investigate the effects of preequilibrium and the gamma strength functions on ${ }^{186} \mathrm{~W},{ }^{107} \mathrm{Ag}$ and ${ }^{93} \mathrm{Nb}$. The calculated results have been discussed and compared with the experimental data.
\end{abstract}

DOI: 10.12693/APhysPolA.132.1076

PACS/topics: 24.10.-i, 25.20.- -x

\section{Introduction}

Because gamma-emission may generally accompany any nuclear reaction, it is one of the most universal channels of the nuclear decay. This process is described in the many-body systems by the radiative strength functions, as well as the absorption of the gamma-rays and the electron-positron decay. These functions are important for lots of studies. Nuclear structure models, decay mechanisms, deformation and fluctuation of the nuclear shape, energies and widths of the collective excitations are good examples from these studies.

Besides this fundamental importance, from the theoretical point of view, the strength functions are necessary for generation of the data for the energy and non-energy applications. It is critically important to have a simple closed-form expression for the $\gamma$-ray strength function, because in most cases this function is used as an auxiliary quantity for calculations of different nuclear characteristics and processes. The theory-based approaches for $\gamma$-ray strength function are preferred over the empirical ones, to improve the reliability and accuracy of such calculations and to understand the physical sense of the used parameters [1].

The nuclear $\gamma$-ray strength function is one of the indispensable inputs needed for reaction-rate calculations, and is particularly important for the neutron-capture cross section [2]. In some studies, only cross sections of photoneutrons and photoprotons have been investigated [3-14]. At the same time, theoretical cross sections have been calculated with TALYS 1.6 in some other studies [15-17].

Gamma strength function describes the (average) energy distribution of photon emission from highlyexcited states or cross section of photon absorption (principle of detailed balance) [18].

*corresponding author; e-mail: nurdankarpuz@amasya.edu.tr

\section{Theoretical method and results}

In this study, the theoretical photoneutron cross sections of ${ }^{186} \mathrm{~W},{ }^{107} \mathrm{Ag}$ and ${ }^{93} \mathrm{Nb}$ in photon-induced reactions have been calculated using TALYS $1.6[19,20]$ nuclear calculations. The calculated results have been compared with experimental data existing in the EXFOR [21] database.

TALYS is a nuclear reaction simulation computer code system for the analysis and prediction of nuclear reactions. The basic objective behind its construction is the simulation of nuclear reactions that involve neutrons, photons, protons, deuterons, tritons, ${ }^{3} \mathrm{He}$ and alphaparticles. TALYS integrates the optical model, direct, preequilibrium, fission and statistical nuclear reaction models in one calculation scheme and gives a prediction for all open reaction channels.

There are five models (Kopecky-Uhl generalized Lorentzian, Brink-Axel Lorentzian, Hartree-Fock BCS tables, Hartree-Fock-Bogolyubov tables, Goriely's hybrid model) for E1 gamma-ray strength function in TALYS. Calculations of theoretical cross section are based on these gamma-ray strength function models [20]. Current study was performed for energy range of the giant dipole resonance region (GDR).

\subsection{Gamma strength functions and equations in literature}

The gamma-ray strength function is related to the photon transmission coefficient by:

$$
T_{X L}^{\gamma}(\varepsilon)=2 \pi \varepsilon^{2 L+1} f_{X L}^{\gamma}(\varepsilon),
$$

because $T_{X L}^{\gamma}$ is directly dependent on $f_{X L}^{\gamma}(\varepsilon)$ the normalization of $f_{X L}^{\gamma}(\varepsilon)$ is of prime importance to the photon transmission coefficient [22].

When normalizing the gamma-ray strength function, there are several ways to model the strength function. We will consider five models, which are the Blatt-Weisskopf method, Brink-Axel Lorentzian, a Kopecky-Uhl generalized Lorentzian, the Hartree-Fock BCS tables, and the Hartree-Fock-Bogolyubov tables [22]. 


\subsubsection{Blatt-Weisskopf}

Use of the Blatt-Weisskopf is $f_{E 1}=$ const [22]. The electric dipole (E1) response of nuclei is dominated by the GDR, a highly excited collective mode above the particle emission threshold [23]. While at higher order levels, E2, M2, etc., the model of Blatt-Weisskopf may be sufficient for $f_{E 2}, f_{M 2}$ and so on, it is not sufficient at $f_{E 1}[22]$.

\subsubsection{Brink-Axel Lorentzian}

The Brink-Axel model describes the standard Lorentzian form of the transmission coefficient at GDR and is calculated as:

$$
f_{X l}\left(E_{\gamma}\right)=K_{X l} \frac{\sigma_{X l} E_{\gamma} \Gamma_{X l}^{2}}{\left(E_{\gamma}^{2}-E_{X l}^{2}\right)^{2}+E_{\gamma}^{2} \Gamma_{X l}^{2}},
$$

where $\sigma_{X l}$ is the strength of the dipole resonance, $E_{X l}$ is the energy, and $\Gamma_{X l}$ is the width of the dipole resonance. $K_{X l}$ is

$$
K_{X l}=\frac{1}{(2 l+1) \pi^{2} \hbar^{2} c^{2}} .
$$

The Brink-Axel Lorentzian is commonly used for all other transmissions, with the exception of M1 [22].

\subsubsection{Kopecky-Uhl generalized Lorantzian}

The general way in which many models depict the photon transmission for $\mathrm{E} 1$ radiation is through the use of a Kopecky-Uhl generalized Lorantzian. It is calculated as:

$$
\begin{aligned}
& f_{E 1}\left(E_{\gamma}, T\right)=K_{E 1}\left[\frac{E_{\gamma} \Gamma_{E 1}\left(E_{\gamma}\right)}{\left(E_{\gamma}^{2}-E_{E 1}^{2}\right)^{2}+E_{\gamma}^{2} \Gamma_{E 1}\left(E_{\gamma}\right)^{2}}\right. \\
& \left.+\frac{0.7 \Gamma_{E 1} 4 \pi^{2} T^{2}}{E_{E 1}^{3}}\right] \sigma_{E 1} \Gamma_{E 1},
\end{aligned}
$$

where $\sigma_{E 1}, \Gamma_{E 1}$ and $E_{E 1}$ are the peak, width, and energy of the giant dipole resonance of the nucleus in question. $T$ is the nuclear temperature:

$$
T=\sqrt{\frac{E_{n}+S_{n}-\Delta-E_{\gamma}}{a\left(S_{n}\right)}},
$$

$E_{n}$ is the incident neutron energy, $S_{n}$ is the neutron separation energy, and $\Delta$ is the pairing connection [19].

2.1.4. Hartree-Fock BCS $\&$ Hartree-Fock-Bogolyubov tables

The last two ways of modeling the E1 strength function allow two microscopic corrections [19]. Using the Hartree- Fock method the E1 strength function is calculated by:

$$
f_{L}\left(E, E_{i}\right)=\frac{2}{\pi} \frac{\Gamma_{\mathrm{GDR}} / 2 E^{2}}{\left.\left(E^{2}-E_{i}^{2}\right)^{2}+\left(\Gamma_{\mathrm{GDR}} / 2\right)^{2} E^{2}\right)},
$$

where $\Gamma_{\mathrm{GDR}}$ is the width of the giant dipole resonance. It is obtained from experimental data when experimental data is available, otherwise it is obtained for empirical statistics.

Using the BCS tables with the Hartree-Fock method or combination of Hartree-Fock with the Bogolyubov method lead to sight perturbations in the calculations of the transmission coefficient, however, as will be seen in the data, the perturbations are small [22].

\subsection{Gamma strength functions in TALYS}

The topic of this work is the influence of the photon strength functions on photonuclear cross sections. These entities, called also $\gamma$-ray or radiative strength functions, have been introduced to describe the process of the emission of photons from highly excited atomic nuclei, that arise from non-relativistic nuclear reactions [24].

Gamma-ray transmission coefficients are important for the description of the gamma emission channel in nuclear reactions [19].

The gamma-ray transmission coefficient for multipolarity $l$ of type $X$ (where $X=M$ or $E$ ) is given by

$$
T_{X l}\left(E_{\gamma}\right)=2 \pi f_{X l}\left(E_{\gamma}\right) E_{\gamma}^{2 l+1},
$$

where $E_{\gamma}$ denotes the gamma energy and $f_{X l}\left(E_{\gamma}\right)$ is the energy-dependent gamma-ray strength function.

The total cross section curves, obtained from photoneutron production for ${ }^{93} \mathrm{Nb},{ }^{107} \mathrm{Ag}$ and ${ }^{186} \mathrm{~W}$ target nuclei are shown in Figs. 1-3. The contribution of gamma strength functions to photoneutron cross-sections were also considered in the target nuclei.

For all transitions, other than E1, the systematic formulae compiled by Kopecky, are used for the resonance parameters. For E1 transitions, TALYS uses [19]

$$
\begin{aligned}
\sigma_{E 1} & =1.2 \times 120 N Z /\left(A \pi \Gamma_{E 1}\right) \mathrm{mb}, \\
E_{E 1} & =31.2 A^{-1 / 3}+20.6 A^{-1 / 6} \mathrm{MeV}, \\
\Gamma_{E 1} & =0.026 E_{E 1}^{1.91} \mathrm{MeV} .
\end{aligned}
$$

For E2 transitions TALYS uses [19]

$$
\begin{aligned}
\sigma_{E 2} & =0.00014 Z^{2} E_{E 2} /\left(A^{1 / 3} \Gamma_{E 2}\right) \mathrm{mb}, \\
E_{E 2} & =63 . A^{-1 / 3} \mathrm{MeV} \\
\Gamma_{E 2} & =6.11-0.012 A \mathrm{MeV} .
\end{aligned}
$$

For multipole radiation higher than E2, TALYS uses [19]

$$
\begin{aligned}
& \sigma_{E l}=8.10^{-4} \sigma_{E(l-1)}, \\
& E_{E l}=E_{E(l-1)} \Gamma_{E l}=x \Gamma_{E(l-1)} .
\end{aligned}
$$

For M1 transitions TALYS uses [19]

$$
\begin{aligned}
& f_{M 1}=1.58 A^{0.47} \text { at } 7 \mathrm{MeV}, \\
& E_{M 1}=41 . A^{-1 / 3} \mathrm{MeV}, \\
& \Gamma_{M 1}=4 \mathrm{MeV} .
\end{aligned}
$$

For multipole radiation higher than M1, TALYS uses [19]

$$
\begin{aligned}
\sigma_{M l} & =8 \times 10^{-4} \sigma_{M(l-1)}, \\
E_{M l} & =E_{M(l-1)}, \\
\Gamma_{M l} & =\Gamma_{M(l-1)} .
\end{aligned}
$$

There are also two microscopic options for E1 radiation. Stephane Goriely calculated gamma-ray strength functions according to the Hartree-Fock BCS model and the Hartree-Fock-Bogolyubov model [19]. 


\section{Conclusions}

In the present work, we have calculated photoneutron cross section with effect of gamma strength functions. The calculated results are compared with experimentally measured data [25-27], taken from EXFOR [21] library. The calculated $(\mathrm{p}, \mathrm{n})$ cross section results for ${ }^{93} \mathrm{Nb},{ }^{107} \mathrm{Ag}$, ${ }^{186} \mathrm{~W}$ target nuclei are shown in Figs. 1-3, respectively.

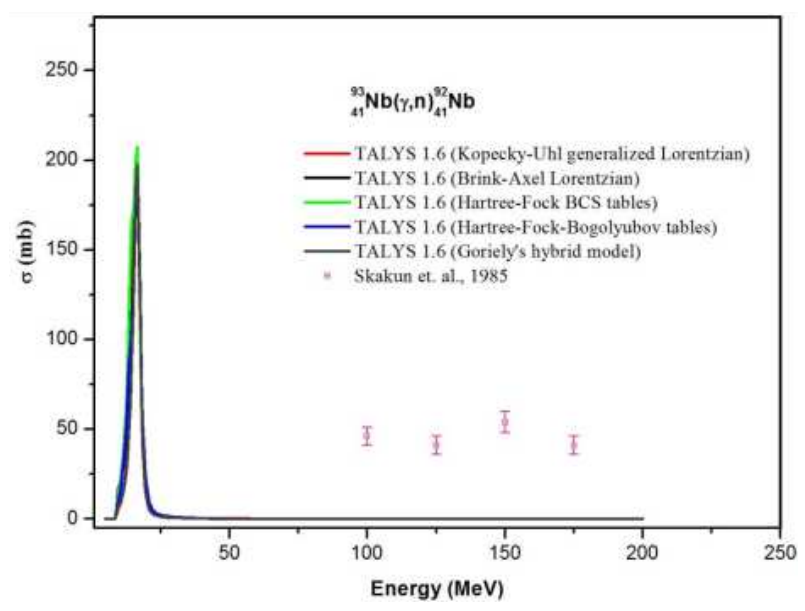

Fig. 1. Comparison between calculated photoneutron cross sections (solid line) and experimental data of Skakun et al. (symbols) for ${ }^{93} \mathrm{Nb}$.

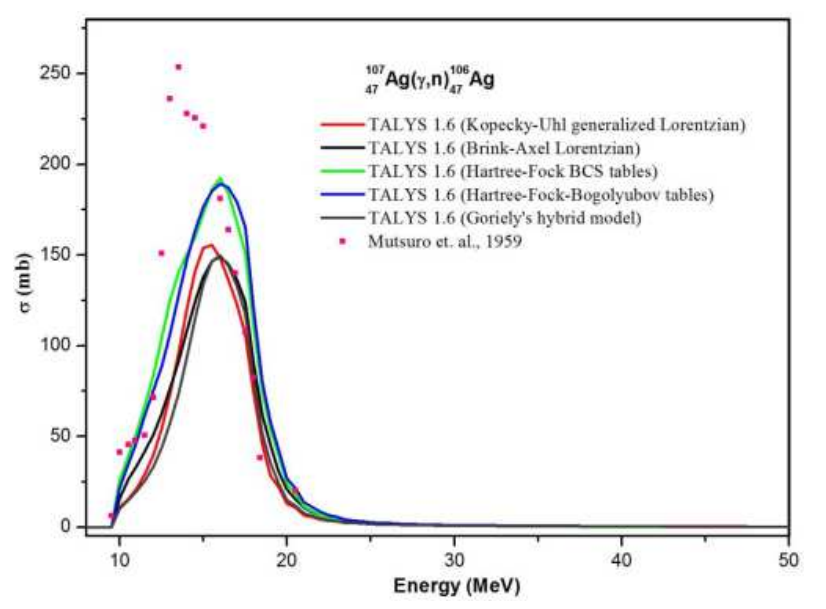

Fig. 2. Comparison between calculated photoneutron cross sections (solid line) and experimental data of Mutsuro et al. (symbols) for ${ }^{107} \mathrm{Ag}$.

In the calculations, all of the gamma strength functions of Kopecky-Uhl generalized Lorentzian, Brink-Axel Lorentzian, Hartree-Fock BCS tables, Hartree-FockBogolyubov tables and Goriely's hybrid model have been used. The effects of the gamma strength function on the cross section exchange data have determined the most compatible type of model. The results, especially for ${ }^{107} \mathrm{Ag}$ and ${ }^{186} \mathrm{~W}$, are in good agreement with the experimental data [25-27] from the EXFOR [17] database.

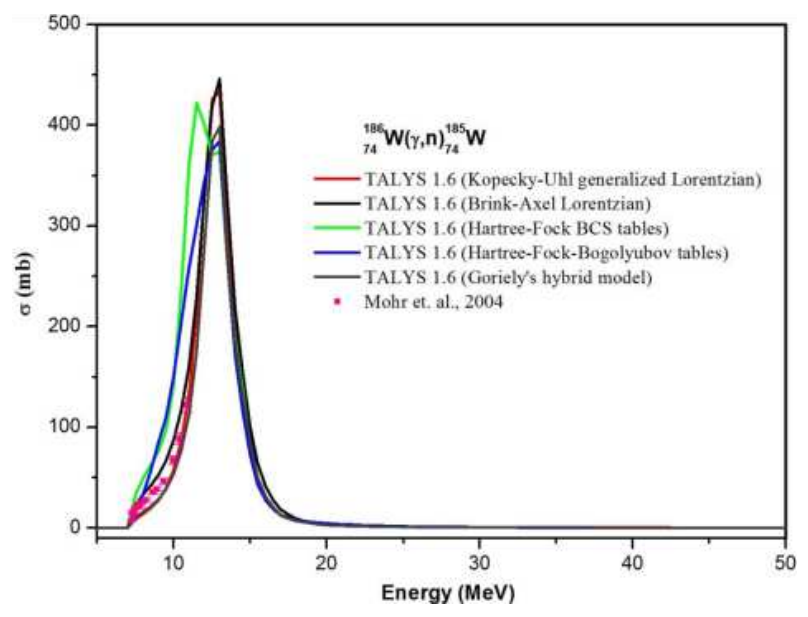

Fig. 3. Comparison between calculated photoneutron cross sections (solid line) and experimental data of Mohr et al. (symbols) for ${ }^{186} \mathrm{~W}$.

The theoretical cross sections of reactions, obtained using the TALYS 1.6 models, clearly demonstrate the GDR effect. According to the calculated theoretical results, Brink-Axel Lorentzian model is the most coherent model.

Good agreement between calculations and the experimental values indicates that TALYS can produce these cross sections without any adjustment of the parameters.

\section{Acknowledgments}

This work has been supported by the Amasya University Foundation Unit (FMB-BAP 15-0132)

\section{References}

[1] V. Plujko, Testing and improvements of gamma-ray strength functions for nuclear model calculations of nuclear data Institute for Nuclear Research, IAEA Contract No. 10308/RO, A Progress Report, Kiev, Ukraine 1998-1999.

[2] A.C. Larsen, S. Goriely, E. Algin, U. Agvaanluvsan, A. Burger, A. Gorgen, M. Guttormsen, T.W. Hagen, T. Lonnroth, G.E. Mitchell, H.T. Nyhus, J.B. Rekstad, T. Renstrom, S.J. Rose, I.E. Ruud, A. Schiller, S. Siem, N.U.H. Syed, H.K. Toft, G.M. Tveten, A. Voinov, K. Wikan. AIP Conf. Proc. 1377, 239 (2010).

[3] N. Karpuz, M.C. Boz, B. Cetin, I. Akkurt, Acta Phys. Pol. A 128, B-411 (2015).

[4] N. Karpuz, M.C. Boz, B. Cetin, I. Akkurt, Acta Phys. Pol. A 128, B-409 (2015).

[5] I. Akkurt, N. Karpuz, B. Cetin, N. Demir, Ann. Nucl. Energy 65, 181 (2014).

[6] A. Kaplan, H. Ozdogan, A. Aydin, E. Tel, Phys. Atomic Nuclei 77, 1371 (2014).

[7] A. Kaplan, I.H. Sarpün, A. Aydin, E. Tel, V. Capali, H. Ozdogan, Phys. Atomic Nuclei 78, 53 (2015). 
[8] A. Kaplan, H. Ozdogan, A. Aydin, E. Tel, Phys. Atomic Nuclei 77, 1371 (2014).

[9] A. Kaplan, H. Ozdogan, A. Aydin, E. Tel, J. Fusion Energy 32, 344 (2013).

[10] A. Kaplan, H. Ozdogan, A. Aydin, E. Tel, J. Fusion Energy 32, 431 (2013).

[11] A. Kaplan, V. Capall, J. Fusion Energy 33, 299 (2014)

[12] A. Kaplan, I.H. Sarpün, A. Aydin, E. Tel, V. Capalı, H. Ozdogan, Phys. Atomic Nuclei 78, 53 (2015).

[13] A. Kaplan, H. Ozdoğan, A. Aydin, E. Tel, Phys. Atomic Nuclei 77, 1371 (2014).

[14] I. Akkurt, J.O. Adler, J.R.M. Annand, F. Fasolo, K. Hansen, L. Isaksson, M. Karlsson, P. Lilja, M. Lundin, B. Nilsson, Phys. Med. Biol. 48, 3345 (2003).

[15] S.S. Noori, N. Karpuz, I. Akkurt, Acta Phys. Pol. A 130, 484 (2016).

[16] N. Karpuz, B. Mavi, I. Akkurt, Acta Phys. Pol. A 130, 313 (2016)

[17] N. Karpuz, M.C. Boz, B. Mavi, F. Oner, I. Akkurt, Acta Phys. Pol. A 128, B-363 (2015).

[18] D. Martin, Do we understand gamma strength functions? The case of ${ }^{96} \mathrm{Mo}$, Institutfür Kernphysik Technische Universitat Darmstadt, (2012). Available on: inis.iaea.org/search/search.aspx, ref. No. 45081928 .
[19] A. Koning, S. Hilaire, S. Goriely, TALYS-1.6 A Nuclear Reaction Program, User Manual, NRG, The Netherlands 2013.

[20] N. Karpuz, B. Mavi, I. Akkurt, Acta Phys. Pol. A 128, B-414 (2015)

[21] EXFOR/CSISRS, Experimental Nuclear Reaction Data File, Brookhaven National Laboratory, National Nuclear Data Center 2009.

[22] B.J.T. Starks, The effect of the E1 strength function on neutron capture cross sections, BrighamYoung University-Idaho, 2009.

[23] Peter von Neumann-Cosel, J. Phys.: Conf. Ser. 337, 012004 (2012).

[24] M. Krticka, Ph.D. Thesis, Charles University in Prague, 2002.

[25] P. Mohr, T. Shizuma, H. Ueda, S. Goko, Makinaga, K.Y. Hara, T. Hayakawa, Y.W. Lui, H. Ohgaki, H. Utsunomiya, Phys. Rev. C 69, 032801 (2004).

[26] N. Mutsuro, Y. Ohnuki, K. Sato, M. Kimura, J. Phys. Soc. Jpn. 14, 1649 (1959).

[27] E.A. Skakun, V.G. Batiy, Yu.V. Vladimirov, Yu.N. Rakivnenko, Yu.N. Ranyuk, O.A. Rastrepin, Izv. Akad. Nauk SSSR, Ser. Fiz. 49, 2252 (1985). 